\title{
El programa lógico de Vaz Ferreira: ¿reforma o revolución?
}

\author{
JOSÉ SEOANE \\ Universidad de la República, Uruguay \\ Sistema Nacional de Investigadores
}

Resumen: El programa encarnado en Lógica Viva supone un antagonismo en relación al tratamiento tradicional de los sofismas. Podría entenderse tal oposición como crítica extensional: el enfoque tradicional solo considera algunos paralogismos, pero hay otros que no tomó en cuenta y es necesario hacerlo. También puede entendérselo en clave intensional: se rechaza la forma tradicional de caracterización de los paralogismos. En el primer caso, la dimensión positiva del programa vazferreiriano operaría por adición; en el segundo, operaría por reconfiguración del campo. ¿Cuál de estas alternativas puede atribuirse a Lógica Viva? Este artículo analiza dos perspectivas de la crítica de Vaz Ferreira: la de Paladino y la de Seoane. La conclusión principal es que ambas perspectivas (con diferencias relevantes) estimulan la alternativa revolucionaria.

Palabras clave: falacias, argumentación, lógica informal, Lógica Viva, Vaz Ferreira. 


\title{
The Logical Program of Vaz Ferreira: Reform or Revolution?
}

\begin{abstract}
Lógica Viva's program exhibits an antagonism in relation to the traditional treatment of sophisms. This opposition could be understood as extensional criticism: the traditional approach only considers some paralogisms, but there are others. Nevertheless, it can also be understood as an intensional criticism: the traditional form of characterization of paralogisms is rejected. In the first case, the positive dimension of Vaz Ferreira's program would operate by addition; in the second, it would operate by reconfiguration of the field. Which of these alternatives can be attributed to Lógica Viva? This paper analyzes two perspectives related to Vaz Ferreira's criticism: those of Paladino and Seoane. The main conclusion is that both perspectives (with relevant differences) stimulate the revolutionary alternative.
\end{abstract}

Key-words: fallacies, argumentation, informal logic, Lógica Viva, Vaz Ferreira.

\section{Introducción}

— s evidente que el programa encarnado en Lógica Viva (en adelante:

$\smile L V)$ supone un cierto antagonismo en relación al tratamiento tradicional de los sofismas. Podría pensarse que aquel desacuerdo consiste, en una primera aproximación, en una suerte de diagnóstico de insuficiencia extensional: el enfoque tradicional considera apenas algunos paralogismos, sin embargo hay otros que no tomó en cuenta y es necesario reparar en ellos. Pero también puede entenderse la oposición vazferreiriana al enfoque clásico de una forma alternativa. La misma se sustentaría en una divergencia intensional: no se trataría, exclusivamente, de promover la atención hacia nuevos tipos de falacias, sino, en general, hacia la forma o modalidad de caracterización de las mismas. Y, más específicamente, la crítica consistiría en el rechazo frontal a la estrategia tradicional de identificación de los paralogismos. Si se acepta la primera opción, el programa positivo de Vaz debiera orientarse a subsanar el déficit extensional: $L V$ debería proponer una expansión del elenco de tipos de razonamientos paralogísticos. Desde esta perspectiva, su aporte se cifraría en el enriquecimiento de la tipología elaborada a través del tratamiento clásico; $L V$ operaría, por así decirlo, por adición. Si se acepta la segunda alternativa, tal objetivo no podría ser el principal. La labor de $L V$ no consistiría, primordialmente, en una ampliación de tipos de sofismas, sino en una revisión de todo el campo; operaría, expresándolo en forma metafórica, 
por reconfiguración. ¿Cuál de estas alternativas puede atribuirse al programa lógico vazferreiriano? En esta nota se analizan dos puntos de vista acerca de la crítica de Vaz a la teoría tradicional de los sofismas: Paladino (1962) y Seoane (2003) y (2015). La conjetura principal de estas páginas es que ambas perspectivas (con diferencias importantes) estimulan una comprensión de dicho programa más próxima a la alternativa "revolucionaria" que a la "reformista". Este resultado, por su parte, auspicia algunas relevantes cuestiones interpretativas. Entre ellas sobresalen tres interrogantes mayores: ¿cuál es el alcance de la crítica vazferreiriana al enfoque tradicional de las falacias?, ¿cómo entender la "complementariedad" de $L V$ respecto de la teoría lógica tradicional? y, especialmente, ¿cómo se relacionan en la propuesta positiva vazferreiriana esquemas argumentales (tradicionalmente) correctos e incorrectos con sus respectivas instancias?

El plan de este artículo es el siguiente. En la sección 2, se realiza un breve comentario histórico, referido a Stuart Mill y su introducción de un nuevo tipo de falacias, a saber, las falacias de confusión. Este motivo funciona, en cierta forma, como articulador de la interpretación de Paladino de la crítica vazferreiriana (sección 3). La sección 4 resume una comprensión alternativa de dicha crítica, cuyo objetivo es desarrollar el esfuerzo de Vaz a los efectos de auspiciar un programa positivo fecundo de tratamiento de los paralogismos. La última sección se propone señalar, a la luz de esta discusión, algunas cuestiones interpretativas abiertas.

\section{Un excurso histórico: John Stuart Mill}

Como es bien conocido, en Mill la reflexión lógica alcanza tanto a la deducción como a la inducción. El Libro II de su monumental obra, A System of Logic, se refiere al primer asunto, los libros III y IV corresponden al segundo. Sus esfuerzos se orientan a caracterizar la aplicación adecuada de ambos tipos de inferencia. El LibroV se dedica a la cuestión de la inferencia errónea, a los "sofismas en general". Podría pues decirse que A System of Logic comprende una parte "positiva" (¿cómo procurar el buen razonamiento?) y una parte "negativa” (¿cómo evitar el mal razonamiento?). Suele recurrirse, para resumir tal actitud, a una cita de este autor:"La filosofía del razonamiento debe, para ser completa, comprender la teoría del mal razonar como la del bien razonar". (Mill 1882: 896; 1917: 723).

El libro V, ciertamente, merecería un análisis detenido, ya que sugiere un original elenco de cuestiones relacionadas con la caracterización y el tratamiento de las falacias. En esta nota, se presentarán, en forma algo grosera, meramente algunos aspectos que se vinculan directamente a la génesis, pro- 
puesta por Paladino, de la posición de Vaz. Identifica así aquel autor el principal aporte de Mill al estudio de las falacias:

Con verdadera profundidad [Mill] observó que, además de los casos en que se razona mal clara y distintamente, hay estados confusos. Y, más aún, hasta sospechó que, en el fondo, todos los sofismas pueden afectar esa forma, pero esa sospecha no fue, en realidad, nada más que una declaración meramente nominal. En su estudio, los sofismas por confusión o de prueba indistintamente concebidas, aparecen como una clase de las falacias; la confusión es el distintivo de un género definido de sofismas. (Paladino 1962: 6)

Merece señalarse que Mill introduce diversas variantes y matices en relación con la tradición de estudios sobre la falacia. Por ejemplo, en línea con su concepción del alcance de los estudios lógicos, amplía el tipo de raciocinio incorrecto a considerar, incorporando a la discusión no exclusivamente el error deductivo, sino también ciertos errores inductivos. Escribe Mill:

Y mientras que los demás autores que no comprenden en su teoría del razonamiento más que la forma silogística, se limitan, conforme a esta limitación, al examen de los sofismas inherentes a esta forma del procedimiento de investigación, nosotros, que queremos tratar del procedimiento entero, debemos añadir a las instrucciones necesarias para hacerlo bien la indicación de las precauciones a tomar para no hacerlo mal en algunas de sus partes; ya peque del lado experimental o del lado racional, ya que el razonamiento y la inducción falten juntamente. (Mill 1882: 897; 1917: 724 ss)

En definitiva, luego de una serie de precisiones, Mill especifica así el objeto de su estudio:

Así, pues, el examen de diversas especies de evidencia puramente aparente, de pruebas concluyentes en apariencia, pero no en realidad, será el objeto de la parte de nuestra investigación en la cual vamos a entrar. (Mill 1882: 900; 1917: 727)

Reaparece en este pasaje la idea central de la vieja definición de cuño aristotélico. "Un argumento falaz, como dicen prácticamente todas las exposiciones desde Aristóteles, es un argumento que parece válido pero no lo es" (Hamblin 2016: 19). Dada la atención de este filósofo a las falacias propias de la inducción, su noción de corrección no coincide con la de validez deductiva. Mill se propone luego enfrentar la tarea de la clasificación de las falacias. El filósofo entiende que las evidencias o las pruebas o las demostra- 
ciones que pueden asumirse equivocadamente en respaldo de las tesis son infinitas. En sus términos, tomadas aquellas sin más especificación, no son susceptibles de clasificación. Es decir, en tanto calificadas exclusivamente por la carencia o negación de una propiedad (a saber: no ser prueba, no ser demostración, no ser evidencia), la clasificación sería imposible. Podría decirse: las cosas que, simplemente, no son pruebas, no admiten clasificación. Pero si se le agrega la propiedad positiva (simular serlo) la situación varía.

Pero las cosas que, no siendo pruebas, son susceptibles de ser tomadas por tales, admiten una clasificación fundada en la propiedad que tienen de simular la prueba. Se las puede clasificar tomando a voluntad, por principio, ya las causas que las hacen parecer pruebas, aunque no lo sean, ya la especie particular de evidencia que simulan. La clasificación de los sofismas, que trataremos de hacer en el siguiente capítulo, está fundada sobre estas dos consideraciones a la vez. (Mill 1882: 901; 1917: 724)

El proceso de construcción de la clasificación posee interés en sí mismo, pero solo se tomará aquí el final de dicho proceso. Este puede resumirse así (donde "sof." está por "sofisma"):

de simple inspección

Sofismas
1. sof. a priori

2. sof. de observación

inductivos

3. sof. de generalización

de prueba

distintamente

concebida

deductivos 4. sof. de razonamiento

de inferencia

de prueba

5. sof. de confusión

no distintamente

concebida 
Como se ha señalado, Mill piensa que la lógica se concentra en la prueba; sobre la misma cabe atender a la observación, deducción y generalización y, consecuentemente, se construye su clasificación de las falacias ${ }^{1}$. De las cinco clases de falacias propuestas por el filósofo, tres de ellas poseen -como señala Vega Reñón- una "clara constitución inferencial”, a saber: las inductivas, vinculadas a la observación y generalización, y las deductivas "representadas paradigmáticamente por los razonamientos que violan la regulación del silogismo" (Vega Reñón 2013: 235). Vega señala la situación diferente, especialmente, en relación a las falacias de simple inspección o a priori "que no parecen envolver de suyo inferencias o pruebas" (Vega Reñón 2013: 235). Se desea llamar la atención del lector acerca de las falacias de confusión, pues se basan en un criterio clasificatorio original: el que permite distinguir entre pruebas distintamente concebidas y pruebas no distintamente concebidas. En relación a las primeras, se puede poseer una percepción clara de la prueba y, sin embargo, errar. El error puede radicar en la (mala) calidad de las premisas o en la (mala) calidad de la inferencia. La calidad de las premisas no queda fuera de la consideración del análisis lógico; quizá es esta una consecuencia natural (en la perspectiva de Mill) de la ampliación del campo de interés argumental i.e. no solo la deducción, sino también la inducción. Pero, el punto importante a retener es que, en tal opción de la clasificación, se posee una representación adecuada (en términos de decodificación) de la prueba o evidencia: esta es "distintamente concebida”. La otra alternativa exhibida por la clasificación es, precisamente, cuando no se da este último rasgo:

Pero puede haber un caso más frecuente aún, que es aquel en que el error proviene de que no se conciben las premisas con bastante claridad, es decir (como hemos visto en el libro precedente), precisión, encontrándose nuestra concepción de la prueba, cuando la aplicamos, distinta de la que era cuando la habíamos formado o admitido, sustituyéndose por inadvertencia, y, en general, inconscientemente, a medida que avanzamos, otras premisas a las primeramente puestas, u otra conclusión a la que quisimos probar primeramente. De aquí una clase de sofismas que pueden ser justamente llamados sofismas de confusión, los cuales comprenden, entre otros, todos aquellos que tienen su fuente en el lenguaje, ya por lo vago o ambiguo de los términos, ya por las asociaciones de ideas que los términos pueden accidentalmente hacer nacer.(Mill 1882: 903ss; 1917: 730 ss)

${ }^{1}$ Véase Hansen y Pinto 1995: 17. 
Se trata pues de identificar la causa del error. ¿Cuál es tal causa? La falta de claridad y precisión que se manifiesta en el uso de las premisas, generador de diversas confusiones en la práctica razonadora. En general, se produce una dinámica conducente al equívoco: se asumen inconscientemente premisas y conclusiones, se varían las mismas... En definitiva, se pierde el control del raciocinio. Esa situación es resultado, entre otros factores, de ciertas propiedades del lenguaje: su ambigüedad y su poder de generar "accidentalmente" asociaciones de ideas equivocadas. Pero el fenómeno es más amplio. Como se advierte, Mill introduce tales sofismas de confusión como una clase o un tipo en su clasificación. Es una suerte de "cajón de sastre... en el que hallan acomodo algunas falacias tradicionales de especial renombre" (Vega Reñón 2013: 233). Por la importancia que posee dicha clase en relación a la reflexión vazferreiriana merecen atención algunos aspectos históricos acerca de su derrotero. ${ }^{2}$

Paladino entiende (siguiendo a Vaz) que el tratamiento tradicional de los sofismas restringe su atención a los mismos como fenómeno expreso, explícito. ${ }^{3}$ El filósofo inglés habría evidenciado luego las limitaciones de esta concepción, a través de su idea de los sofismas de pruebas indistintamente concebidas -adviértase que algunas estrategias falaces tradicionalmente identificadas caen bajo esta nueva categoría-. ${ }^{4}$ No obstante, no habría logrado liberarse totalmente de la influencia del punto de vista tradicional; esta liberación llegaría con Vaz. La siguiente extensa cita de Paladino permite captar esta idea:

Sin embargo, John Stuart Mill -como lo comprendemos después de la Lógica viva- malogró, en parte, una idea importante. Pudo haber dado un alcance mucho mayor a su concepto del sofisma de confusión, considerando que no solamente la ambigüedad de términos, la petición de principios y la ignorancia de la cuestión sino todos los sofismas pueden presentar la modalidad de ser confusamente pensados. Como todavía, entre esos dos tipos extremos, clara o confusamente concebidos, hay que tener en cuenta las gradaciones intermedias, se comprende que existe, en realidad, una gama prácticamente infinita de modos de incurrir en los sofismas... (Paladino 1962: 7)

\footnotetext{
${ }^{2}$ Es altamente recomendable el capítulo dedicado a Mill de Vega Reñón (2013), especialmente merece destacarse la atención que presta a la "historia" de las falacias de confusión, incorporando el esfuerzo de Vaz en esa estela.

${ }^{3}$ Vaz Ferreira explícitamente afirma esto, por ejemplo, en el capítulo "Valor y uso del razonamiento" de Lógica Viva; véase Vaz Ferreira 2008: 190.

${ }^{4}$ Véase Hansen y Pinto 1995: 17ss.
} 


\section{La crítica vazferreiriana: una interpretación}

T as observaciones sobre Mill que elabora Paladino son agudas.

Efectivamente Mill opta por los beneficios de la clasificación y deja de lado algunas intuiciones que aparecían más o menos implícitas en sus desarrollos. Como elegante y comprensiblemente señala el intérprete, Mill habría "sospechado" que "todos los sofismas" podrían entenderse como sofismas de confusión. Pero, finalmente, habría optado por confinarlos a una clase. Esta es la opción que (entiende Paladino) supera originalmente Vaz Ferreira; en cierta forma, Lógica Viva vendría a radicalizar la "sospecha" de Mill y asumir la certeza de una generalidad mucho mayor del fenómeno detectado por el filósofo inglés. La idea central de Paladino es que la teoría tradicional de los sofismas estaría gobernada por un supuesto inconsciente central: "las falacias se producen siempre clara y distintamente concebidas" (Paladino 1962: 5). Usando la terminología vazferreiriana, agrega Paladino "que todos los sofismas son "expresos, discursivos". Mill habría intuido la falsedad de este supuesto; Vaz habría asumido claramente su negación. El fundamento profundo de tal rechazo radicaría en una nueva concepción de la actividad psicológica, alejada de los prejuicios atomistas.

¿Cuál es el apoyo textual más claro que ofrece Paladino para atribuir a Vaz tales convicciones? Un complejo y enigmático pasaje de "Un paralogismo de actualidad": 5

Mucho hizo John Stuart Mill por corregir esta tendencia, con su estudio de los sofismas de pruebas indistintamente concebidas; pero creo que se equivocó al suponer que las falacias de confusión eran una clase de falacias; más bien, y ya que es fuerza establecer esas clases, refiriendo a ficticios esquemas típicos nuestros falsos razonamientos como lo hacemos con los buenos, más bien hay que presentar las falacias de confusión, no como una clase de falacias sino como un modo de caer en las falacias, sea cual sea la clase. De manera que habrá diversos modos de caer en las falacias: sin razonar, o casi sin razonar...; razonando muy confusamente, menos confusamente, y así por grados hasta el caso en verdad menos común del mal raciocinio distintamente concebido... (Vaz Ferreira 1938: 131-132)

\footnotetext{
${ }^{5}$ No se pretende insinuar que se trate del único apoyo textual para respaldar la interpretación de Paladino. Por el contrario, su estudio es competente y profundo y, sin dudas, puede encontrar un rico respaldo en la obra de Vaz. Solo se pretende señalar la importancia del pasaje en cuestión. Sobre este trabajo de Paladino puede resultar útil consultar la breve pero informativa reseña de Claps 1963.
}

JOSÉ SEOANE - El programa lógico de Vaz Ferreira: ¿reforma o revolución? I 245-265 
El texto ofrece diversos desafíos. Pero conviene en este momento destacar una nota fundamental: las falacias de confusión, más que constituir una clase, describen un "modo de caer" en el raciocinio falaz, "sea cual sea su clase". Fiel a su línea interpretativa, Paladino extrae, esencialmente, tres conclusiones del análisis de este iluminador pasaje. En primer lugar, la perspectiva tradicional identifica los paralogismos a través de "esquemas típicos", desconociendo la especificidad de aquellos. Cada sofisma (entiende Paladino qua intérprete deVaz) es "único, diferente en cada caso". Dicho en forma sintética: la clasificación sistemática es, simplemente, "imposible". Pero, además, tales clasificaciones no pueden ser "rigurosas". Con un lenguaje conjuntístico, podría decirse que no generan una auténtica partición del universo. Pues, en segundo lugar, estas clases se intersectan, se “compenetran". Además, dada la extraordinaria multiplicidad de grados que admite la comisión del sofisma, su número crece exponencialmente. Es decir, cada sofisma supone la posibilidad de una extraordinaria cantidad de variantes, correspondientes a los diferentes grados o matices referidos porVaz. Más que tipos de falacias, Vaz llamaría la atención sobre la diversidad extraordinaria de "modos de caer" en los paralogismos. En esta línea interpretativa, la crítica vazferreiriana dejaría escasísimo (si algún) margen para la apelación al esquema como recurso metodológico. ¿Es este un resultado inusual o extravagante? No. Al contrario, parece corresponder a una línea más bien dominante en la comprensión de tal crítica. Así, por ejemplo, Pereda (con su habitual fineza y erudición) nos enseña la posibilidad de distinguir, en el tratamiento de las falacias, dos grandes tradiciones: la lógico-dialéctica o aristotélica y la naturalista o baconiana. ${ }^{6}$ A riesgo de simplificar mucho el contraste, podría quizá decirse que la primera identifica las falacias con "esquemas de argumentar incorrecto", mientras la segunda lo hace con "mecanismos de argumentar incorrecto". Pereda parece no dudar acerca de en cuál tradición ubicar a Vaz (y, consecuentemente, cuál es su descendencia):

Independientemente de la clasificación de los ídolos, a partir de Bacon se han sucedido muchos intentos de reconstruir mecanismos que nos llevan a producir argumentos falaces. Así, Vaz Ferreira estudia mecanismos falaces como los que producen falsa oposición, falsa precisión, o que nos llevan a confundir cuestiones de palabras con cuestiones de hechos. Por otra parte, en los últimos tiempos, a partir de esta actitud baconiana o naturalista se han desarrollado importantes investigaciones empíricas sobre el razonamiento. Estas conforman

\footnotetext{
${ }^{6}$ Véase Pereda 2011: 249-252.
} 
materiales de enorme valor para complementar y discutir no solo las teorías sobre los diversos mecanismos falaces, propias de la tradición naturalista, sino también las teorías de las falacias en tanto esquemas de argumentar inválido, propias de la tradición lógico-dialéctica. (Pereda 2011: 252-253)

Vega Reñón parece inclinarse por una comprensión análoga, al entender que las ideas de Vaz:

[...] se oponen a la pretensión clásica del análisis lógico de atenerse al razonamiento expreso, que es por cierto una directriz congruente con la concepción tradicional de los sofismas como falacias clara y distintamente concebidas. Frente a esta idea de sofisma, los paralogismos que a Vaz Ferreira le importa detectar y examinar vienen a ser procesos por los que caemos o nos encontramos en estados de neblina que tiñen, velan o enturbian nuestra mente [...] En otros términos, tampoco muy precisos, cabría hablar no solo de confusiones, sino de disposiciones o predisposiciones ideológicas, y así remontarse a otros precedentes como los ídolos (Bacon) o los prejuicios (Helvetius), o de marcos estereotipados, en la ola del análisis contemporáneo del discurso. (Vega Reñón 2008: 48)

Quizá valga la pena formular el contraste entre el proyecto de Mill y el proyecto de Vaz en términos algo diferentes a aquellos planteados por $\mathrm{Pa}-$ ladino. La perspectiva de Mill parece entenderse más bien como enfrentada a la posición tradicional en términos de una crítica fundamentalmente extensional: el punto de vista tradicional solamente habría atendido a aquellos sofismas de pruebas distintamente concebidas. Se trataría entonces de considerar los casos en que el sofisma se produce, no por error en la manipulación de la información a través de la prueba, sino por una codificación o decodificación defectuosa de la misma, es decir, por "indistinción" de la prueba. En tal sentido, aunque los "géneros" incluidos en la clase de falacias de confusión no sean novedosos, tal clase se vuelve extensionalmente más hospitalaria en la medida en que incorporaría un enriquecido espectro de variantes de aquellos esquemas, debido a las múltiples formas de emergencia de la confusión. La superación de esta insuficiencia se lograría luego a través de una estrategia aditiva: súmese a aquellas clases de falacias tradicionales, una nueva, a saber, la falacia de confusión, que captura los casos en que las pruebas no son distintamente concebidas.

Una radicalización de ese esfuerzo se encontraría en el proyecto de Vaz. Para este autor el punto no sería entender el fenómeno de las pruebas no distintamente concebidas tanto como determinante de una nueva clase, sino como un "modo de caer" en cualquier clase de falacia. Tales modos además conforman (como se señaló) una infinidad impresionante de grados 
o matices. Luego el problema para Vaz no radicaría en una insuficiencia extensional, sino resultaría más bien en una cuestión de naturaleza intensional: la definición de clases de falacias es impropia, pues no es capaz de captar aquella relevante diversidad. La estrategia superadora de la insuficiencia anotada, propia del punto de vista tradicional, luego, no puede ser aditiva (no se trata de sumar clases, a los efectos de completar la clasificación), sino que lo que se requiere es una revisión, reformulación o reconfiguración global del campo de estudio. Si se explota la metáfora política del título de este ensayo, Mill sería un reformista, Vaz un revolucionario.

Paladino avanza su opinión acerca de la razón profunda de este eminente contraste. El fundamento radicaría, dicho de una forma directa, en la existencia de dos actitudes diversas respecto a la capacidad del lenguaje de captar el pensamiento. Para Mill las pruebas no indistintamente concebidas eran, en última instancia, reformulables en términos claros y distintos. Para Vaz, tal tarea es, en muchos casos, simplemente imposible. Paladino expone así el dilema:

En efecto, hay dos maneras de entender lo confuso, lo subdiscursivo: como convertible al discurso, como un discurso informulado, por una parte; como irreductible al discurso, por otra. (Paladino 1962: 9)

Entendidas así las alternativas disponibles, la distribución de los papeles resulta previsible: Mill (fiel a la "vieja psicología atomista”) opta por la primera opción; Vaz (apoyado en James y Bergson), por la segunda. La "lógica tradicional" reduciría las falacias a "esquemas verbales" -dominada por la convicción del carácter expreso (actual o potencial) de todo sofisma-. Y la innovación de Lógica Viva consistiría en

[...] estudiar por primera vez los sofismas reales. En la gran mayoría de los casos estos no son expresos sino confusos y de tal índole de confusión que no tiene sentido pensar en reconstruir discursivamente el falso razonamiento. De la antigua psicología que confundía los procesos mentales con el discurso, era víctima la lógica. (Paladino 1962: 10)

\section{La crítica vazferreiriana: una interpretación/desarrollo}

Ta descripción que emerge de la discusión de Paladino del pro-

grama vazferreiriano es sólida, perfectamente argumentada y exhibe un apoyo textual estimable. En contraposición, el programa vazferreiriano así caracterizado parece fenecer, una vez enunciado. La posibilidad 
de progresar en su desarrollo lógico resulta prácticamente imposible, pues no se evidencian herramientas o alternativas generales que sugieran hacia dónde puede evolucionar. Por supuesto, podría pensarse que su futuro se apoye, exclusivamente, en el campo psicológico; de hecho, esa perspectiva es la que sugiere Pereda en el pasaje arriba citado. ${ }^{7}$ No obstante, el programa vazferreiriano, desde un punto de vista lógico amplio, deja traslucir un conjunto de intuiciones metaargumentales valiosas que reclaman razonadamente ser tenidas en cuenta. ¿Qué hacer?

Un texto filosófico puede interpretarse o desarrollarse. Interpretar un texto filosófico supone procurar descifrar su significado, identificar sus tesis y aislar sus argumentos. Desarrollar un texto filosófico, en cambio, consiste en seleccionar ciertas ideas fundamentales, consideradas valiosas, aunque necesitadas o susceptibles de afinamiento, y lograr su mejor formulación. El objetivo fundamental de una interpretación es alcanzar la máxima fidelidad al original; la aspiración principal de un desarrollo es proveer mayor precisión y fecundidad a aquellas ideas esenciales. ${ }^{8}$ En trabajos anteriores (Seoane 2003, 2008 y 2015) se fue perfilando una lectura de la crítica vazferreiriana, con voluntad quizá más de desarrollar que de interpretar las ideas del filósofo.

¿Cuál es esa lectura? Resumidamente, la crítica de Vaz poseería tres niveles: pragmático, ideológico, metodológico. El primero refiere a la impotencia del tratamiento tradicional para captar los paralogismos más relevantes en la práctica argumental; la lógica no contribuiría sustantivamente a prevenir el error y mejorar la calidad de la argumentación. El segundo apunta a una especie de trabajo negativo "extra" del análisis tradicional: no solo no permite tratar los paralogismos más comunes, sino que genera una visión distorsionada de la realidad argumental, concentrando la atención en el nivel estructural (sintáctico, gramatical...), cuando el partido se juega en el plano semántico-pragmático. El tercer nivel es el más decisivo: el enfoque tradicional es impotente y distorsionador, por su opción metodológica. El problema central de dicho enfoque es pues la metodología. Un diagrama permite ilustrar las relaciones entre estos niveles, partiendo desde el más básico:

\footnotetext{
${ }^{7}$ ¿Confirma la idea de Pereda trabajos tales como los desarrollados por D. Kahneman y A. Tversky? Se espera responder a esta cuestión en un futuro ensayo. El lector interesado en aquellos enfoques puede consultar, por ejemplo, Kahneman 2011 o una revisión reciente de "ilusiones cognitivas" en Pohl 2017.

8 Esta caracterización de la distinción interpretación/desarrollo es literalmente la ofrecida en Seoane 2019.
} 


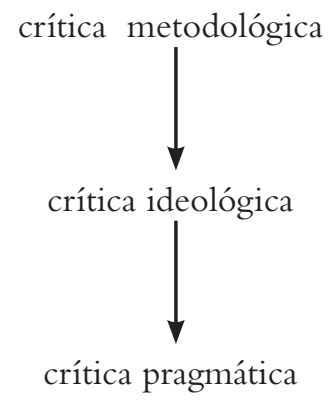

¿Existe algún apoyo textual que respalde esta interpretación? La dimensión pragmática (la impotencia práctica de las prevenciones lógicas tradicionales) parece manifestarse aquí:?

Presentado así, el paralogismo es tan simple y fácil de evitar, que no parece importante escribir al respecto [...] Pero esa impresión es la que sentimos generalmente cuando se nos presenta el esquema lógico de cualquier falacia; otra cosa muy distinta es librarse en la realidad, y en todo momento, de caer en falacias de las que, simplificadas, tendrían ese esquema por expresión lógica [...].Cuando estudiamos en los tratados lo que es una petición de principios o un círculo vicioso, nos parece inconcebible que en estado de salud mental se pueda incurrir en tales falacias. Hasta la ambigüedad de términos y la ignorancia de la cuestión, nos parecen causa de error que cualquier persona de juicio medianamente recto podría evitar con un poco de atención; $y$, entre tanto,... (Vaz Ferreira 1938: 130-131; itálicas J. S.)

El siguiente pasaje puede tal vez respaldar la dimensión ideológica: ${ }^{10}$

Observaciones de orden teórico concernientes a las relaciones de la psicología y la lógica, del pensamiento y el lenguaje, etcétera, destinadas a corregir los conceptos falsos que el esquematismo de la lógica ha originado. (Vaz Ferreira 2008: 36; itálicas J. S.)

El pasaje siguiente apoya las consideraciones pragmáticas y, en particular, el decidido énfasis metodológico:

\footnotetext{
${ }^{9}$ Los pasajes reproducidos aquí de Fermentario pertenecen a un artículo titulado "Un paralogismo de actualidad" (incluido el citado por Paladino); el mismo aparece ya publicado en 1908 en Carlos Vaz Ferreira 1 (Montevideo: Mariño y Caballero).

${ }^{10}$ Como el lector recuerda, la primera edición de Lógica Viva se remonta a 1910.
} 
Sería un estudio de la manera como los hombres piensan, discuten, aciertan o se equivocan -sobre todo de la manera como se equivocan- pero de hecho: un análisis de las confusiones más comunes, de los paralogismos más frecuentes en la práctica, tales como son, no tales como serían si los procesos psicológicos fueran superponibles a sus esquemas verbales... (Vaz Ferreira 2008: 35; itálicas J. S.)

Y, más específicamente, puede advertirse el subrayado metodológico en el pasaje siguiente:

Lo que hay es que esos tratados, o nuestra manera de entenderlos, nos hacen pensar predominantemente en las falacias, no como son en la realidad psicológica, sino como serían si el que incurre en ellas hiciera el mal raciocinio de una manera clara, expresa, discursiva. (Vaz Ferreira 1938: 131; itálicas J.S.)

¿Cuál es el problema metodológico que desencamina el punto de vista tradicional? Asumir que "los procesos psicológicos" siempre son "superponibles a sus esquemas verbales" o, dicho de otra forma, asumir que siempre "el mal raciocinio" se presenta "de una manera clara, expresa, discursiva". Por supuesto, pueden existir diversas formas de entender el "método" erróneo. Quizá podría pensarse que Paladino tiende a identificarlo con la, en principio, siempre viable explicitación plena. Otro modo de entender la crítica de Vaz (que es el que se defenderá aquí) identifica su blanco en forma neta: el método objetado consistiría en la caracterización de todas las clases argumentales relevantes a través de sus respectivos esquemas formales o estructurales. Captar una falacia se reduciría así, en la perspectiva estándar, a ofrecer su "retrato" estructural. Adviértase que el fundamento de tal repulsa no necesariamente debe ser el onerosamente asumido porVaz, a saber, la relación lenguaje-pensamiento (que es la opción de Paladino). Podría simplemente asumirse que, a los efectos de revelar la falla metodológica tradicional, alcanzaría con mostrar casos donde fracase notoriamente su capacidad teórica o sistemática. Bastaría, por así decir, mostrar fenómenos paralogísticos paradigmática o ejemplarmente "rebeldes" a aquella metodología, y evidenciar, empíricamente, la generalidad del fenómeno. ${ }^{11}$ Esta

${ }^{11}$ En general, la eficacia del reduccionismo esquemático en algunos casos, no implica su eficacia para cualquier caso. Una revisión de la certidumbre acrítica respecto del éxito de la metodología sintactista en el contexto lógico puede leerse en Etchemendy 1983. En cierta forma, este último texto inspiró algunos aspectos de la lectura de la crítica de Vaz desarrollada en esta sección. 
podría considerarse una de las funciones a cumplir por los casos estudiados en Lógica Viva.

Más específicamente, dado que la teoría lógica es una teoría de segundo orden, debe representar los objetos de primer orden: los argumentos y sus propiedades. Y, dado que hace ambas tareas a la luz de aquella metodología defectuosa, mal representa los argumentos y mal representa su naturaleza paralogística. Es decir, la crítica de Vaz supondría entonces este doble rechazo (metodológico) al punto de vista tradicional. Así entendida su dimensión crítica, la dimensión positiva del programa de Vaz debiera, precisamente, superar ambos problemas: el de la representación de los argumentos y el de la evaluación de su condición paralogística. A diferencia del enfoque de Paladino, la perspectiva propuesta identifica, en forma precisa, la metodología objetada con una metodología básica perfectamente atribuible a la teoría lógica, libera el fundamento de la crítica del compromiso acerca de la insuficiencia epistémica radical del lenguaje y discrimina su impacto en una dimensión doble: representacional y evaluativa. Algunos aspectos de este enfoque cómodamente se pueden ubicar como interpretativos; otros parecen merecer más bien la calificación de desarrollos.

Pero hay aún una nota más a rescatar del esfuerzo crítico vazferreiriano. Existe un aspecto en el pasaje de Fermentario que inteligentemente seleccionó Paladino para respaldar su interpretación que resulta muy difícil desatender. La expresión en cuestión es la siguiente: “[...] y ya que es fuerza establecer esas clases, refiriendo a ficticios esquemas típicos nuestros falsos razonamientos como lo hacemos con los buenos [...]" (Vaz Ferreira 1938: 131)

¿Qué pretende significarVaz con esta compleja aseveración? Paladino ciertamente no propone una interpretación para la misma. Pero quizá valga la pena ensayar una conjetura. En primer lugar, pareciera implicar que no hay otra alternativa, para proseguir el estudio de las falacias, que la construcción de clases argumentales.Vaz afirma que "es fuerza establecer esas clases". Pero, además, la construcción de las mismas se asocia a la definición o caracterización esquemática. En palabras de Vaz: "refiriendo a ficticios esquemas típicos nuestros falsos razonamientos". Y, en tercer lugar, esta estrategia de especificación de clases no es exclusiva del tratamiento tradicional de los paralogismos; por el contrario, el método es exactamente el mismo que se emplea cuando se trata de los buenos razonamientos. Es decir, es forzoso definir clases, a partir de esquemas típicos, en el caso de los "falsos razonamientos como to hacemos con los buenos".

Esta apelación a los esquemas no debería resultar ajena a la perspectiva vazferreiriana. Cuando el filósofo examina distintos tipos de clasificaciones (en el capítulo de $L V$ titulado "Psicología y lógica de las clasificaciones y falacias verbo-ideológicas relacionadas") distingue aquellas que proporcionan 
límites precisos y criterios de selección netos (las habituales de la matemática elemental), algunas donde ya los límites no son tan precisos y los criterios menos claros (algunos casos de la biología) y aquellas como, por ejemplo, las referidas a las enfermedades mentales, donde los criterios pierden notablemente precisión y rigor. Respecto de estos últimos casos, señala tres actitudes posibles. Hay dos que considera abiertamente inadecuadas: aquella que consiste en "tomar las clasificaciones vagas como si fueran clasificaciones precisas" y aquella que se reduce "a concluir que las clasificaciones no sirven". La actitud preconizada porVaz, respecto de las clasificaciones en tales contextos, es "tomarlas como lo que son, a saber: como esquemas para pensar, para describir, para enseñar y hasta para facilitar la observación" (LV: 183). La admisión razonada de los esquemas parece así configurarse como la opción adecuada.

Pero, entonces, ¿cómo conciliar tal comprensión con la crítica del filósofo a la teoría tradicional de los sofismas? Quizá pueda conjeturarse que el blanco de la crítica es el reduccionismo esquemático, no toda apelación a esquemas o estructuras lingüísticas. Esta última observación supone incluir una nueva diferencia importante con el enfoque de Paladino.

Así entendida la crítica, el programa positivo vazferreiriano bien podría suponer alguna forma de combinar el recurso estructural con otras herramientas de análisis. Retomando el contraste introducido por Pereda entre la tradición lógico-dialéctica o aristotélica y la tradición baconiana o naturalista de tratamiento del raciocinio falaz, merece destacarse que la perspectiva esbozada ubicaría quizá a Vaz en algún punto entre ambas tradiciones, algo más próximo a la primera. ${ }^{12}$ Esta idea de combinar o mixturar recursos de diversa naturaleza guio la elaboración del modelo $M$, un intento de desarrollar la metodología alternativa implícita en $L V .^{13}$

$M$ es pues un modelo "mixto". Apela a un componente estructural o formal (sintáctico, en un sentido amplio) y a un componente no formal (semántico y pragmático). El análisis de los paralogismos operaría así a través de la identificación de una clase argumental abierta, difusa. Aproximarse a tal clase supondría desarrollar la capacidad de advertir un parecido de familia entre sus componentes. La adquisición de tal capacidad devendría de la exposición a una serie de casos ordenados y adecuadamente estudiados. Ese esfuerzo es el que, en cierta forma, sintetiza o encarna $M$ (para cada caso particular de falacia). Por ejemplo, si se tratara de la falsa oposición, se podría

${ }^{12}$ Así entendido el programa vazferreiriano, parece que debiera contarse como precedente de esfuerzos posteriores tales como aquellos que se centran en el concepto de "esquema argumental" (véase, por ejemplo, Walton, Reed y Macagno 2008).

${ }^{13}$ La primera versión del modelo M aparece en Seoane 2003; la última, en Seoane 2019. 
captar el componente formal a través de los recursos de la lógica de primer orden y debiera apelarse al análisis semántico-pragmático para responder, fundamentalmente, a las siguientes cuestiones: ¿hay una relación de oposición entre los predicados relevantes?, si no la hay, ¿cuál es la relación que hay entre ellos?, ¿cómo puede descifrarse lo subdiscursivo a partir del contexto? Como se advierte, el componente formal juega un papel en la adquisición conceptual de la falacia, pero no la caracteriza en términos de instanciación; sin la apelación al componente no formal es imposible adquirir el concepto y, consecuentemente, entender si se está o no frente a un caso paralogístico. En síntesis, se trata de usar esquemas y construir clases de una forma alternativa a la forma tradicional. Parece dificil atribuir sin más el modelo $M$ a Vaz Ferreira (qua interpretación) pero, a su vez, parece robustamente justificado entenderlo como haciendo justicia a la dimensión crítica y a la dimensión positiva del proyecto vazferreiriano (es decir, qua desarrollo).

Si las conclusiones anteriores son aceptables, parece plausible atribuir a Vaz una actitud más bien revolucionaria que reformista. Los ejemplos de $L V$ cumplirían así una doble función. Evidenciar instancias del fracaso metodológico tradicional e instruir sobre una metodología novedosa. Por lo tanto, no parece justificado limitar la superioridad de tal metodología, exclusivamente, a estos casos de paralogismos; todos los tipos de falacias pareciera que debieran merecer un tratamiento innovador análogo.Y si, tal cual dice Vaz, el recurso esquemático ha sido ensayado para la argumentación falaz al igual que para la argumentación correcta, ¿cuáles debieran ser las fronteras de la revolución?

\section{5. ¿Reforma o revolución?}

Tal vez valga la pena formular (desprejuiciadamente) una pregunta crucial: ¿cuál es el alcance de la crítica expuesta (fundamentalmente) en $L V$ ? O, si se prefiere, ¿cuál es el alcance pretendido de la innovación metodológica delineada por Vaz? Se han bosquejado aquí dos respuestas posibles. Dicho de una forma esquemática: reforma o revolución. Nótese que optar por una perspectiva reformista o revolucionaria del programa vazferreiriano posee un impacto obvio en la comprensión del carácter "complementario" de $L V$ respecto del tratamiento tradicional de las falacias y, en general, de la teoría lógica. Si se elige la primera perspectiva, entonces tal complementariedad supondría entender $L V$ como (para usar la expresión de Vaz) un "segundo libro" que prolonga el contenido del primero, a través de un método novedoso. Si no se encuentran ambos mundos incomunicados, parecería que la comunicación (en la mejor hipótesis) solo podría 
suponer una dirección: desde la perspectiva tradicional a la vazferreiriana. Si se prefiere, en cambio, la segunda perspectiva, la complementariedad debiera comprenderse en un sentido abiertamente bidireccional: la teoría lógica podría prestar valiosos servicios al proyecto vazferreiriano pero, a su vez, este impactaría notablemente en la comprensión de aquella. En el segundo pasaje arriba citado de Vaz Ferreira 1908, el filósofo parece ubicar la falla metodológica en los tratados tradicionales o en "nuestra manera de entenderlos". Este matiz abre una tentadora posibilidad: apreciar el proyecto vazferreiriano como una nueva forma de entender la teoría lógica tradicional, especialmente comprendida esta como herramienta orientada al incremento en la calidad del debate público.

Si se acepta cualquiera de las dos líneas interpretativas de la crítica metodológica vazferreiriana arriba expuesta, una alternativa debe descartarse: la opción reformista. Es decir, tanto en la esclarecedora interpretación de Paladino como en la sugerida en este ensayo, el blanco de la crítica no se reduciría a un método fallido en relación a ciertos tipos de falacias. Y, consecuentemente, el programa positivo vazferreiriano no podría caracterizarse en términos meramente aditivos: sumar nuevos tipos de paralogismos, tratables con una metodología alternativa "paralela" a la tradicional. La situación pareciera lucir de modo decididamente diverso: no se trata de reformar, sino de revolucionar o reconfigurar. Para decirlo de otra forma: quizá se pudiera entender la política de Mill como extensional y aditiva. La diferente actitud de Vaz en relación al enfoque tradicional, lo llevaría naturalmente a proponer reconfigurar el campo del estudio de las falacias en su totalidad, como resultado de una renovación intensional del concepto de paralogismo. Si tal fuera el territorio a revolucionar, entonces el programa de Vaz supondría una comprensión renovada de todos los tipos o clases de falacias. En ese sentido, Lógica Viva escogería algunos casos novedosos a los efectos de ilustrar el método y operaría a la vez en una doble direccionalidad: extendiendo el territorio por anexión de nuevas provincias (falsa oposición, cuestiones de palabra y cuestiones de hecho) e ilustrando, con su tratamiento, el advenimiento del nuevo régimen para la totalidad del territorio.

Pero, además, corresponde acotar que tal totalidad no es meramente la unión de lo descripto en $L V$ más lo descripto en los tratados tradicionales. La naturaleza programática de la obra y el carácter abierto e inconcluso de este programa resultan evidentes al lector del "Prológo a la primera edición" de Lógica Viva:

[...] lo que concibo no es un libro, sino un tipo de libros que podrían escribirse en número indefinido, porque su materia es inagotable, y siempre serán útiles. (Vaz Ferreira 2008: 35) 
De esta forma, el programa vazferreiriano pareciera sugerir su expansión en dos direcciones: sobre los conocidos territorios del tratamiento tradicional de las falacias, promoviendo la comprensión de las mismas en una clave novedosa, y sobre los desconocidos territorios aún inexplorados, emergentes de un incesante desplazamiento de las fronteras, ya que la "materia" que concierne a estos estudios "es inagotable".

Definido el carácter revolucionario del programa vazferreiriano, aún resta contestar una pregunta especialmente relevante: ¿cuál es, estrictamente, el territorio a revolucionar? Vaz señala que es forzoso apelar a la clasificación en el caso de los "malos razonamientos" así "como lo hacemos con los buenos". Luego, si la apelación a la estrategia puramente esquemática resulta insuficiente para aplicar la lógica a la argumentación falaz, ¿tal déficit no impacta en la argumentación correcta? O, puesto de otra forma, ¿la aproximación esquemática es insuficiente (si se trata del paralogismo) y suficiente (si se trata de la argumentación correcta)? ¿No debieran extenderse las fronteras de la revolución a todo el estudio del razonamiento? Quizá, desde la perspectiva vazferreiriana, a la hora de articular lógica y práctica argumental, resultara necesario abandonar en general la estrategia puramente esquemática -tal cual sugiere la comprensión de la crítica vazferreiriana defendida en estas páginas-. En otras palabras: el territorio a revolucionar debiera ser la totalidad del campo lógico.

Un enfoque de tal audacia estimula una comprensión renovada de los esquemas argumentales correctos y, más específicamente, de las relaciones entre argumentación correcta y argumentación falaz. Desde tal perspectiva, ciertos esquemas (tradicionalmente considerados falaces) son susceptibles de ser "instanciados" por argumentos falaces así como por argumentaciones correctas. ${ }^{14}$ Pero, si la línea interpretativa propuesta del programa vazferreiriano es correcta, también tendríamos casos (por ejemplo, las denominadas falacias verbo-ideológicas) de esquemas argumentales (tradicionalmente considerados correctos) susceptibles de ser "instanciados" por argumentaciones correctas así como por argumentaciones falaces. Tres cuestiones importantes se han debatido en estos últimos párrafos: ¿cuál es el alcance de la crítica vazferreiriana al enfoque tradicional de las falacias?, ¿cómo entender el papel "complementario" de $L V$ respecto de la teoría lógica tradicional? y, especialmente, ¿cómo se relacionan, en la propuesta positiva vazferreiriana, esquemas argumentales e instancias correctas e incorrectas? Como advierte el lector, un estudio minucioso y profundo de tales interrogantes excede largamente

${ }^{14}$ Puede consultarse al respecto, por ejemplo, Tindale 2007 y Gensler 2016. 
los objetivos de este ensayo; luego estas páginas debieran entenderse apenas como estímulo a la investigación futura. ${ }^{15}$

\section{BIBLIOGRAFÍA}

Claps, M. (1963), “Julio Paladino: La Lógica Viva y la teoría de los sofismas”, Cuadernos Uruguayos de Filosofía, II: 186-187.

Etchemendy, J. (1983), “The Doctrine of Logic as Form”, Linguistics and Philosophy, 6: 319-334.

Gensler, H. (2016), Introdução à Lógica (São Paulo: Paulus).

Hamblin, Ch. (2016), Falacias, trad. H. Marraud (Lima: Palestra Editores).

Hansen, H. V. y Pinto, R. C. (1995), Fallacies Classical and Contemporaries Readings (Pennsylvania:The Pennsylvania State University Press).

Kahneman, D. (2011), Thinking, Fast and Slow (New York: Farrar, Strauss and Giroux).

Mill, J. S. (1882), A System of Logic, Ratiocinative and Inductive, $8^{\text {th }}$ ed. (New York: Harper and Brothers).

Mill, J. S. (1917), Sistema de Lógica Inductiva y Deductiva, trad. E. Ovejero y Mauri (Madrid: Daniel Jorro).

Paladino, J. (1962), La lógica viva y la teoría de los sofismas (Montevideo: Universidad de la República).

Pereda, C. (2011), "Falacia”, en L. Vega Reñón y P. Olmos Gómez (2011) (comps.), Compendio de lógica, argumentación y retórica (Madrid:Trotta, 249-252).

Pohl, R. F. (2017) (ed.), Cognitive Illusions: Intriguing Phenomena in Thinking, Judgement and Memory, $2^{\text {nd }}$ ed. (London-New York: Rutledge, Taylor and Francis Group).

Seoane, J. (2003), “Un modelo vazferreiriano de análisis argumental”, Papeles de Trabajo (colección de pre-publicaciones, FHCE, Universidad de la República): 1-17.

Seoane, J. (2008), "Introducción a Lógica viva: ¿es posible desarrollar el análisis argumental vazferreiriano?”, en C. Vaz Ferreira (2008), Sobre lógica: textos de Carlos Vaz Ferreira, (Montevideo: Biblioteca Nacional y Departamento de Publicaciones FHCE, 11-31).

Seoane, J. (2015), "Revisitando Lógica Viva: un modelo de análisis argumental”, Signos Filosóficos, XVII, 34: 58-77.

Seoane, J. (2019), "Falsa oposición: cinco enigmas para el intérprete”, Diánoia, 64: 85-113.

${ }^{15}$ Una elaboración inicial de este trabajo apareció en Versión Cero, un espacio de difusión de pre-publicaciones del Departamento de Lógica y Filosofía de la Lógica, FHCE, UdelaR. Esta versión amplía y modifica sustantivamente aquella primera. Desearía agradecer a Gustavo Pereira y Miguel Andreoli, que me llamaron la atención sobre los aportes de Kahneman y su posible relevancia en relación con el tratamiento vazferreiriano de los paralogismos.

JOSÉ SEOANE - El programa lógico de Vaz Ferreira: ¿reforma o revolución? I 245-265 
Tindale, C. M. (2007), Fallacies and Argument Appraisal (Cambridge: Cambridge University Press).

Vaz Ferreira, C. (1938), Fermentario (Montevideo:Tipología Atlántida).

Vaz Ferreira, C. (2008), Sobre lógica: textos de Carlos Vaz Ferreira (Montevideo: Biblioteca Nacional y Departamento de Publicaciones FHCE).

Vega Reñón, L. (2008), “Sobre paralogismos: ideas para tener en cuenta”, Crítica, Revista Hispanoamericana de Filosofía, 40: 45-65.

Vega Reñón, L. (2013), La fauna de las falacias, (Madrid:Trotta).

Vega Reñón, L. y Olmos Gómez, P. (2011) (comps.), Compendio de lógica, argumentación y retórica (Madrid:Trotta).

Walton, D., Reed, Ch. y Macagno, F. (2008), Argumentation Schemes (Cambridge: Cambridge University Press).

Recibido: 08-03-2019; aceptado: 14-05-2019 